\title{
Broad spectrum antimicrobial PDMS-based biomaterial for catheter fabrication
}

\author{
Arunmozhiarasi Armugam', Siew Ping Teong ${ }^{1}$, Diane S. W. Lim', Shook Pui Chan', Guangshun Yi', Dionis S. Yew², \\ Cyrus W. Beh ${ }^{1,2}$ and Yugen Zhang ${ }^{1 *}$
}

\begin{abstract}
Background: In addition to the widespread use of antibiotics in healthcare settings, the current COVID-19 pandemic has escalated the emergence of antibiotic resistance. Nosocomial infections among hospitalized patients is a leading site for such resistant microbial colonization due to prolonged use of invasive devices and antibiotics in therapies. Invasive medical devices, especially catheters, are prone to infections that could accelerate the development of resistant microbes. Often, catheters - particularly urinary catheters - are prone to high infection rates. Antibiotic-coated catheters can reduce infection rates and although commercially available, are limited in efficacy and choices.

Methods: Herein, a novel and facile method to fabricate PMDS-based biomaterial for the development of antimicrobial eluting catheters is presented. Silicone based organic polymer polydimethylsiloxane (PDMS) was used to prepare a biomaterial containing novel polymeric imidazolium antimicrobial compound.

Results: It was found that the PDMS-based biomaterials could eradicate microbial colonization even after 60 days in culture with continuous microbial challenge, be recycled over multiple uses, stored at room temperature for long-term usage and importantly is biocompatible.
\end{abstract}

Conclusion: The PDMS-based biomaterial displayed biocidal functionality on microbes of clinical origin, which form major threats in hospital acquired infections.

Keywords: Antimicrobial, Composite, PDMS, Catheter, Biomaterial

\section{Introduction}

The COVID-19 pandemic has introduced another wave of inappropriate and excessive utilisation of antibiotics, biocides, and disinfectants that will hamper ongoing worldwide efforts towards the control of antimicrobial resistance. Nosocomial infections have escalated to a new magnitude of emergency in the current pandemic era $[1,2]$ at a time when medical device-associated infections have superseded surgical site infections in healthcare services [3]. As part of this trend, urinary catheter associated infections are becoming increasingly rampant

\footnotetext{
* Correspondence: ygzhang@ibb.a-star.edu.sg

Arunmozhiarasi Armugam is the first author.

${ }^{1}$ Institute of Bioengineering and Bioimaging, 31 Biopolis Way, The Nanos,

Singapore 138669, Singapore

Full list of author information is available at the end of the article
}

in healthcare settings with high mortality and morbidity events [4, 5]. To circumvent device-related infection, several antimicrobial-coated catheters (silver-coated Dover $^{\text {Tm }}$ silicone catheter and Rochester Medical Magic 3 nitrofurazone-coated silicone catheter) have emerged in clinical use, albeit with limited success. The existing products, particularly those relying on metal ions as the antimicrobial component, are subject to the following concerns: (a) limited efficacy; (b) inability to sustain continuous antimicrobial functionality over a prolonged period [6-8] as well as (c) potential toxicity [9]. Thus, there is a pressing need to explore the fabrication of new biomaterials with antimicrobial properties that could address these disadvantages. 
In order to overcome infections related to medical devices, materials for medical devices, especially catheters, are fabricated to contain inherent antimicrobial properties. Two of the widely reported methods for achieving this are surface grafting using functionalized groups and surface coating using antimicrobial compounds $[4,10]$. Though showing encouraging results in the laboratory, materials prepared by surface grafting/functionalization [11] and surface coating [12] procedures often deplete their antimicrobial functionality and efficiency within a short duration. This was observed for the commercially available products. Several studies have also reported on the feasibility of impregnating antimicrobial compounds within the catheter material using the swelling to evaporation method. Peritoneal silicone catheters impregnated with rifampicin, triclosan and trimethoprim inhibited colonization of Methicillin-resistant Staphylococcus aureus (MRSA) for 90 days in a flow model [13]. The authors proposed that the combinatorial antimicrobial impregnated catheter could prove beneficial for long-term clinical applications [14]. Recently, casting a blend of biomaterials with the antimicrobial agents to produce composite biomaterials has been demonstrated [15]. While surfaces that were functionalized, grafted or coated with antimicrobials were observed to only be bacteriostatic, biomaterials that were impregnated or casted with antimicrobials appear to exhibit bactericidal properties [16].

Though various polymers are being used to fabricate a wide range of medical devices, polydimethylsiloxane (PDMS) elastomers have emerged as a promising lead for catheter development. PDMS is innately biocompatible, chemically stable, transparent and mechanically elastic. Nevertheless, being chemically inert, PDMS lacks reactive functional groups on its surface. Pre-treatments such as high-energy treatment or chemical etching are usually required to incorporate useful functional groups for surface modification [16]. On the other hand, impregnation of useful antimicrobials or biocides into commercially available silicone catheters is heavily dependent on the solubility of the antimicrobial compounds in organic solvents $[14,17,18]$. In both cases, the entire process is usually complex and laborious, requiring several sequential stages to remove the solvents used for grafting or impregnation. These laborious protocols are unfavourable and hinder their application in industrial-scale fabrications. In addition, these biomaterials have short shelf lives (durability) and short-lived antimicrobial efficacy. They also require low temperature storage conditions, adding to their production cost.

Herein, a facile and simple method for fabricating PDMS-based biomaterials (PDMS-PIM) exhibiting antimicrobial properties, is reported. Instead of grafting or functionalizing the PDMS surface, we modified a novel amphiphilic main-chain poly-imidazolium (PIM) [19, 20] with a styrene functional groups that react directly with PDMS precursors and integrate to form a functional biomaterial. It is noteworthy that PDMS biomaterials are also used in biomedical applications as medical implants in orthopaedic, dental, cardiovascular, prostheses and as well as in tissue engineering [21]. The release profile indicated an initial burst of antimicrobial compound (PIM), followed by sustained release. Biocidal properties of PDMS-PIM were observed over duration up to 60 days under continuous microbial challenge. In addition, excellent biocidal effect was also demonstrated against several multi drug resistant (MDR) clinical isolates, illustrated the broad spectrum antimicrobial property of the PDMS-PIM biomaterial. The biocidal properties of our biomaterial was observed up to 6 months upon storage at room temperature, thus showing a promising, long shelf life.

\section{Materials and methods}

Microbial strains and cell lines

Microbes, Escherichia coli (E. coli, ATCC 15036), Staphylococcus aureus (S. aureus, ATCC 6538), Candida albicans (C. albicans, ATCC 10231) and murine fibroblast cell line (NCTC clone 929; L929, $\mathrm{ATCC}^{\oplus} \mathrm{CCL1}^{\mathrm{Tm}}$ ) were purchased from American Type Culture Collection (ATCC.org). Klebsiella pneumonia (CI0027), Serratia marcescens (CI0183A1), Pseudomonas aeruginosa (CI0183A2), Acinetobacter baumannii (RI0139A), Enterobacter aerogenes ESBL-sensitive (RI0006A2) Methicillinresistant Staphylococcus aureus (R10309N), Vancomycin resistant Enterococcus faecium (RI0252N1), Candida tropicalis (RI0243A), Candida parapsilosis (ATCC 22019) Candida auris (NCPF 8977) were kind gift of Dr. Shawn Vasoo from National Centre for Infectious Disease, Singapore.

\section{Preparation of PDMS silicone rubber}

PDMS Sylgard ${ }^{\oplus} \mathrm{R} 184$ (Dow Corning Corporation) is a heat curable PDMS supplied as a two-part kit consisting of prepolymer (base) and cross-linker (curing agent) components. The pre-polymer and cross-linker were mixed at a 10:1 weight ratio and cast onto a petri dish (manufacturer's recommendation). The composite was degassed under vacuum to remove air bubbles and processed for curing at $70{ }^{\circ} \mathrm{C}$ overnight. The cured PDMS sheet was cut into square pieces of $2.5 \mathrm{~cm} \times 2.5 \mathrm{~cm}$, with thickness around 1 $\mathrm{mm}$. The PDMS composite materials were then washed with ethanol and dried at $60^{\circ} \mathrm{C}$ before use.

\section{Synthesis of PIM45-vinyl imidazolium antimicrobial polymer}

The synthesis of PIM45-vinyl was carried out between PIM45 with imidazole terminal and 4-vinylbenzyl 
chloride. The imidazolium polymer material, PIM45 was synthesized using the previously reported method [19]. 4-vinylbenzyl chloride (56 mg, $3.0 \mathrm{eq}$ of PIM45) was added to $1.25 \mathrm{~mL}$ EtOH solution containing PIM45 (250 mg, $0.122 \mathrm{mmol}$ ). The mixture (in a sealed vial) was stirred at $65^{\circ} \mathrm{C}$ for $16 \mathrm{~h}$. The suspension was transferred to a $15 \mathrm{~mL}$ centrifuge tube and purified following repetitive precipitation from THF. The compound was then dried under reduced pressure at $90{ }^{\circ} \mathrm{C}$ that resulted in a pale yellow powder of PIM45-vinyl as end product. The PIM45-vinyl synthesis scheme is presented in Fig. S1 and the synthesised derivative was verified by proton nuclear magnetic resonance $\left({ }^{1} \mathrm{H}\right.$ NMR) spectrum as shown in Fig. S5.

\section{Preparation of PIM45-vinyl casted composite PDMS-PIM material.}

PIM45-vinyl (0.11 g, $1 \mathrm{wt} \%)$ in $2 \mathrm{ml}$ THF was mixed thoroughly with PDMS base and curing agent (10:1 ratio) in a mix-cure process. The quasi-solid substance were degassed under vacuum to remove solvent and air bubbles followed by curing at $70{ }^{\circ} \mathrm{C}$ (overnight). The cured PDMS sheet was cut into square pieces of $2.5 \mathrm{~cm} \times 2.5 \mathrm{~cm}$, with thickness around $1 \mathrm{~mm}$. The PDMS samples were rinsed with ethanol and dried at $60{ }^{\circ} \mathrm{C}$ before use.

\section{Characterization of biomaterial surface}

a) Surface wettability measurement.

PDMS based silicone materials are naturally hydrophobic. The static contact angle of PDMS-PIM and pristine PDMS material surfaces were measured on OCA15 contact angle analyzer (Future Digital Scientific Corp., U.S.A.). Deionized (DI) water $(2 \mu \mathrm{L})$ at $3 \mu \mathrm{L} / \mathrm{sec}$, was used for all measurements [17]. All samples were analysed in triplicates, and the static contact angle data were presented as mean \pm SD. PIM45-vinyl casted composite PDMS material at various weight \% of PIM45vinyl was selected for our study and depicted as PDMSPIMx (where $x$ represents the \% PIM45-vinyl used to formed the composite).

b) Surface roughness assessment.

Topographic images of the PDMS and PDMS-PIM material surfaces were obtained from Nanoscope 9.7 Dimension ICON Atomic Force Microscope (Bruker). PDMS material were rinsed in deionised water and $70 \%$ ethanol before drying them at $60{ }^{\circ} \mathrm{C}$. The dried material was subjected to a jet of compressed air to remove any particles on the surface. AFM tapping mode (phase contrast) measurements were performed with a scanning rate of $0.995 \mathrm{~Hz}$ using a non-contact long reflex (NCLR) probe $(\mathrm{k}=48 \mathrm{~N} / \mathrm{m}, \mathrm{f}=190 \mathrm{kHz})$. For each sample, multiple images from $5 \times 5 \mu \mathrm{m}$ were acquired at 3 different locations per disk. NanoScope Analysis 2.0 software were used to analyse the AFM images to obtain the Mean Roughness $(\mathrm{Ra})$ and the Root Mean Square roughness (Rq). Ra and Rq were calculated and averaged from 3 to 5 identical scan areas $(5 \mu \mathrm{m} \times 5 \mu \mathrm{m})$.

c) Scanning Electron Microscopy (FESEM) of material surface.

The surfaces of the PDMS and PDMS-PIM material was characterized by Field Emission Scanning Electron Microscopy (FESEM, JEOL JSM-7400E). Briefly, the material was washed with water followed by $70 \%$ ethanol and air dried. The dried disk was then coated with thin platinum film using high resolution sputter coater (JEOL, JFC-1600 Auto Fine Coater; coating conditions: $20 \mathrm{~mA}, 30 \mathrm{~s}$ ) and viewed under SEM.

\section{d) Mechanical property of the PDMS and PDMS-PIM material.}

Tensile stress-strain experiments was carried out to characterize the mechanical strength of our PDMS and PDMS-PIM material. The mechanical property of the PDMS materials were evaluated on 3340 Series Single Column Table Frames (INSTRON 3344 universal testing machine (Instron, Norwood, Massachusetts). The 25 $\mathrm{mm} \times 25 \mathrm{~mm}$ dimension PDMS material was clamped to expose $12 \mathrm{~mm}$ midsection of the material. The test samples were mounted on two mechanical grips with one grip attached to the cross head where the load cell is mounted and other grip attached to the fixed end. The initial length of the sample was recorded and subsequently the device stretched the samples, vertically at a constant speed of $10 \mathrm{~mm} / \mathrm{min}$. Data was collected and processed using the respective software. All tests were conducted at room temperature and at least in duplicates for each tested PDMS material (pristine and exposed to microbes). Relationship between the stress and strain, load $(\mathrm{N})$, ultimate tensile strength $(\mathrm{MPa})$, elongation $(\mathrm{mm})$ and modulus $(\mathrm{MPa})$ were determined at end point at maximum elongation.

\section{Characterization of PIM45-vinyl released in solution}

The stability of the antimicrobial compound in the casted composite PDMS material was evaluated by both quantitative and qualitative assessments. All experiments were carried out in at least three replicates.

a) The identity of the compound eluted from the PDMS-PIM material was confirmed by ${ }^{1} \mathrm{H}$ NMR Spectroscopy. Composite PDMS-PIM material (1 
$\mathrm{cm}^{2}$ ) was immersed in $\mathrm{D}_{2} \mathrm{O}$ and incubated at 300 $\mathrm{rpm} /$ room temperature for $1 \mathrm{~h} .{ }^{1} \mathrm{H}$ NMR spectroscopy was then carried out on the $\mathrm{D}_{2} \mathrm{O}$ solution to determine the chemical profile of the released antimicrobial compounds.

b) The concentration of released PIM45-vinyl was determined using spectrophotometric method. UV spectrum scan was carried out for PIM45-vinyl in solution. Maximum absorption $(\lambda \max )$ at $252 \mathrm{~nm}$ was observed for the polymer and subsequently used for the semi-quantitative assay. Briefly, the biomaterial $(100 \mathrm{mg})$ was placed in $10 \mathrm{ml}$ of phosphate-buffered saline (PBS) in $15 \mathrm{ml}$ centrifuge tubes and incubated at $300 \mathrm{rpm}$ at room temperature for $1 \mathrm{~h}$. The PDMS material was sequentially and periodically transferred to a fresh tube containing $10 \mathrm{ml}$ PBS. The resultant PBS solution containing the released antimicrobial was subsequently used for semi-quantitative assay.

c) Antimicrobial activity of the eluates were determined using the GB 15979-2002 protocol and as described by Malcolm et al. [22] Briefly, $10 \mathrm{ml}$ PBS containing the released antimicrobial compounds (from 100 mg of PDMS-PIM material) was inoculated with microbes (bacteria, E. coli and $S$. aureus or fungi, C. albicans) to a final concentration of $10^{4} \mathrm{CFU} / \mathrm{mL}$. The inoculum was incubated at room temperature for $1 \mathrm{~h}$ at $100 \mathrm{rpm}$. The bactericidal and fungicidal efficiencies of the leached compounds were evaluated by the plate assay. The viable colonies was computed for colony forming units per milliliter (CFU/mL) of inoculum.

\section{Surface antimicrobial assay}

The antimicrobial property of the fabricated biomaterial was determined using the Japanese Industrial Standard (JIS Z 2801: 2010) Protocol [23]. Briefly, 100 ul microbial in suspension at $10^{6} \mathrm{CFU} / \mathrm{mL}$ in pure media (TSB or YMB; unless otherwise specified), was applied onto the surface of pristine PDMS or PDMS-PIM material (2.5 $\mathrm{cm} \times 2.5 \mathrm{~cm}$ ) disk. A thin plastic film of $2 \mathrm{~cm} \times 2 \mathrm{~cm}$ was then overlaid on the cell suspension. The disk was incubated at appropriate temperature for $24 \mathrm{~h}$ (standard protocol). Incubation time point was adjusted as deviation from the standard protocol (for end point assays) as deem necessary. The material was then rinsed with $9.9 \mathrm{ml}$ of TSB/YMB and the cell suspension was plated on $\mathrm{LB}$ agar plates. The plates were incubated at $36^{\circ} \mathrm{C}$ for 16-18 $\mathrm{h}$ and viable microbe indicative of colony forming units $(\mathrm{CFU} / \mathrm{mL})$ was computed. Representative microbes, Gram negative (E. coli), Gram positive (S. aureus) and fungi (C. albicans) have been used for the evaluation. All experiments were carried out in three replicates.
Biofilm formation and anti-biofilm assay (time-kill study) Biofilm development was carried out according to Fisher et al. [14] and Merritt et al. [24] with slight modification. Microbes $(100 \mathrm{ul})$ at $10^{6} \mathrm{CFU} / \mathrm{ml}$ in media were applied on sterile glass cover slips in six well plates. The microbial inoculate was allowed to grow and attach itself onto the cover slip for $72 \mathrm{~h}$ to form mature biofilm. The cover slips were then rinsed with PBS to remove unattached, planktonic bacterium, following the growth of biofilm. Formation of the biofilm was confirmed following crystal violet staining. The PDMS-PIM material (in triplicate) were then placed over the biofilm (on cover slips). Following incubation, the bacteria samples were harvested according to Mandakhalikar et al. [25] at intervals of $1 \mathrm{~h}$, $2 \mathrm{~h}, 4 \mathrm{~h}$ and $24 \mathrm{~h}$. Plate assay was carried out to determine colony forming units of viable bacteria. All experiments were carried out in three replicates.

\section{Long-term stability via continuous microbial challenge} The continuous microbial challenge protocol was adapted from Zhang et al. [26] with slight modifications. The experimental protocol is similar to the contact killing assay (JIS Z2801-2010 protocol) albeit with extended incubation period. Briefly, the PDMS and PDMS-PIM disk was sterilized via UV exposure for 30 mins. Microbial suspension at $10^{6}$ cells $(100 \mathrm{ul}$ for $2.5 \mathrm{~cm} \times 2.5 \mathrm{~cm}$ disk) in respective media (TSB for $S$. aureus or YMB for C. albicans or both) was applied onto the PDMS and PDMS-PIM material and covered with thin plastic films $(2 \mathrm{~cm} \times 2 \mathrm{~cm})$. The samples were incubated at $37^{\circ} \mathrm{C}$ for stipulated time points. Bacterial suspension was periodically inoculated/refreshed every $48 \mathrm{~h}-72 \mathrm{~h}$ incubation and continued until the endpoint. At a pre-determined end-point (3 Days, 5 Days, 7 Days, 15 Days, 30 Days, 45 Days and 60 Days), the respective PDMS and PDMSPIM materials were rinsed with $10 \mathrm{ml}$ PBS and plated, following appropriate dilutions to compute for colony forming units $(\mathrm{CFU} / \mathrm{mL})$. All experiments were carried out in three replicates.

\section{Biocompatibilty evaluation of PDMS-PIM biomaterial}

Both red blood cell hemolysis assay and mammalian cell viability assay was used to evaluate the biocompatibility of our PDMS-PIM material [27].

\section{Hemolysis assay}

Fresh blood (mice) was rinsed 5 times in saline $(0.9 \%$ $\mathrm{NaCl})$ at $500 \mathrm{~g} / 5 \mathrm{mins}$ to remove the plasma. The subsequent red blood cells (RBC) was rinsed $2 \mathrm{x}$ in PBS to prepare the working solution. RBC suspension was diluted to $4 \% \mathrm{vol} / \mathrm{vol}$ in PBS for the hemolysis assay. The diluted RBC (100 ul) were added to 96 well plate followed by $100 \mathrm{ul} \mathrm{PBS}$, in the presence of $5 \mathrm{mg}$ or $10 \mathrm{mg}$ PDMSPIM material and incubated at $35^{\circ} \mathrm{C}$ for $1 \mathrm{~h}$. The plates 
were then centrifuged at $2200 \mathrm{rpm} / 5$ mins. An aliquot $(100 \mathrm{ul})$ of the supernatant was transferred to a new 96 well plate. The hemoglobin released upon RBC lysis was determined spectrophotometrically at $576 \mathrm{~nm}$. The percentage of lysis was calculated using TritonX100 treated positive control and untreated control RBC [28]. The data was analyzed and expressed as mean and standard deviation of three replicates for quantification.

\section{Cell viability assay}

Cell viability assay was carried out using the murine derived fibroblast (L929) in culture. L929 murine fibroblast cells were cultured in DMEM complete media (supplemented with $10 \%(\mathrm{v} / \mathrm{v})$ heat-inactivated fetal bovine serum (FBS), $100 \mathrm{U} / \mathrm{ml}$ penicillin and $100 \mu \mathrm{g} / \mathrm{ml}$ streptomycin) at $37{ }^{\circ} \mathrm{C}$ in $100 \%$ humidity and $5 \% \mathrm{CO}_{2}$. Fibroblast cells were seeded at $1.0 \times 10^{4}$ cells/well $(100 \mathrm{ul})$ in 96 well plate for overnight growth. The overnight culture of L929 in 96 well plate were incubated with $5 \mathrm{mg}$ and $10 \mathrm{mg}$ PDMS and PDMS-PIM material. Cell viability assay was carried out using the Alamar Blue reagent according to manufacturer's protocol (Thermofisher Inc). Briefly $10 \mathrm{ul}$ of Alamar Blue Reagent was added to 90 ul of complete DMEM media (DMEM containing 10\% FBS and 1\% Penicillin/Streptomycin) in the wells and incubated for $1-4 \mathrm{~h}$ at room temperature. Fluorescent intensity of viable cells were read at $570 \mathrm{~nm} / 590$ $\mathrm{nm}$. The cell viability was calculated as the ratio of the absorbance of treated cells to the absorbance of the control groups (untreated). All experiments were performed in triplicate in three independent experiments.

\section{Statistical analysis}

Data were expressed as means \pm standard deviation of the mean (S.D.). Student's $t$-test was used to determine significance among groups. A difference with $P<0.05$ was considered statistically significant." $p<0.05$, * $p<$ $0.01,{ }^{* * * *} p<0.001$.

\section{Results}

PDMS is the choice material for a wide range of medical applications, especially for catheter preparation. A number of strategies have been developed for PDMS surface modification to add on additional functions for specific applications. Here, we propose a bulk modification method to make antimicrobial PDMS-based biomaterials instead of device surface modification. With the new PDMS composite materials (PDMS-PIMx, $\mathrm{x}=$ weight percentage of PIM in composites), one can make any device or product based on PDMS with inherent disinfection and antifouling property.

Imidazolium polymer PIM45 is reported with excellent antimicrobial efficacy and the polymer chains are typically ended with imidazole group [19, 20]. Platinum is a good catalyst for the hydrosilylation of styrene and its derivatives [29]. Herein, PIM45-vinyl (Scheme 1, Fig. S1) was casted with PDMS to fabricate a biomaterial (PDMS-PIM). In this study, THF was selected as solvent to mix PIM45-vinyl and PDMS base and curing agent homogenously to proceed the mix-cure process. The quasi-solid substance were degassed under vacuum to remove solvent and air bubbles followed by curing at $70{ }^{\circ} \mathrm{C}$ for overnight. PDMS biomaterials (PDMS-

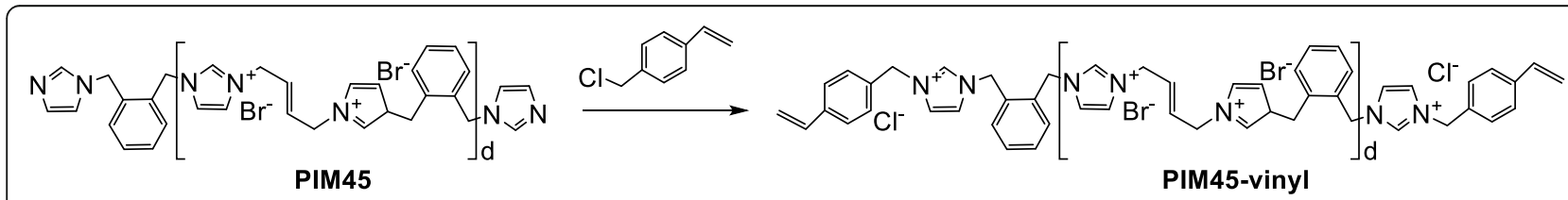

Base<smiles>C=C[Si](C)(C)O[Si](C)(C)O[Si](C)(C)O[Si](C)(C)C=C</smiles>

Curing agent
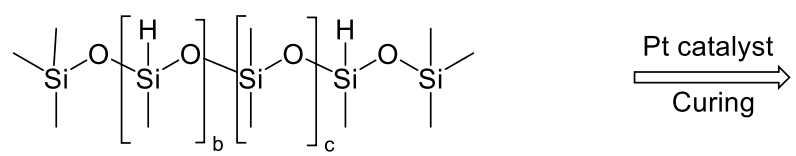

PIM45-vinyl
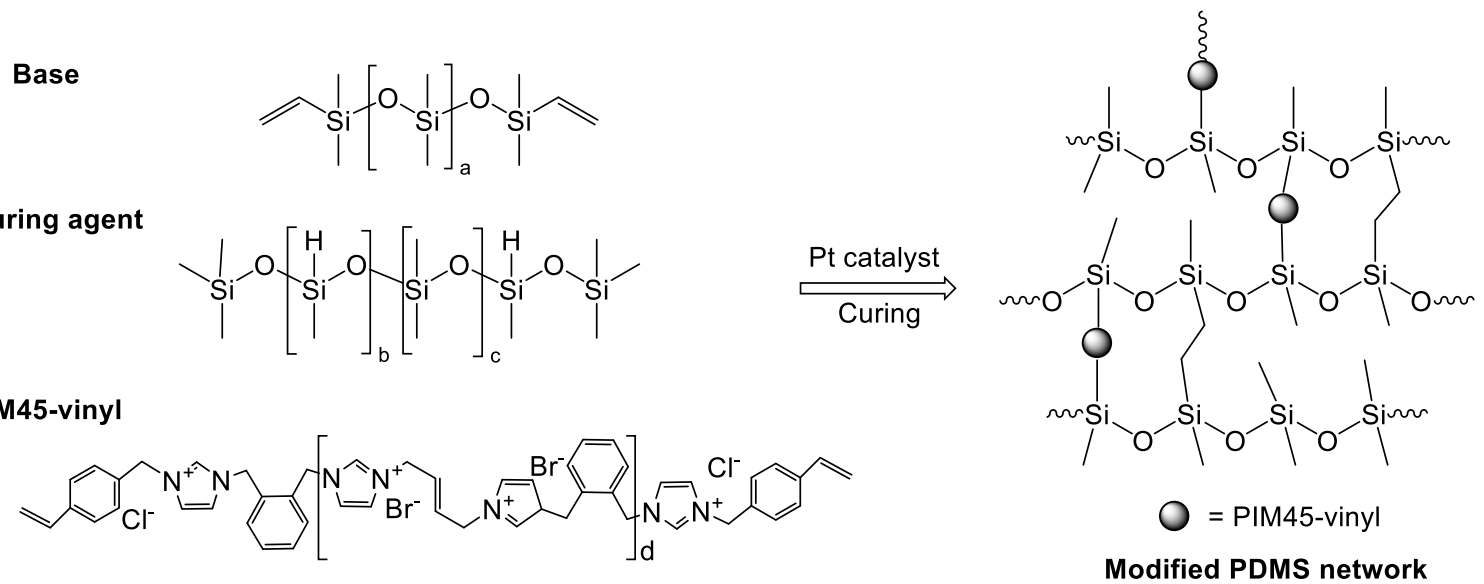

Scheme 1 Preparation of antimicrobial PDMS composite biomaterial 
PIM0.5, 1, 2, 5) with different PIM loadings (0.5 wt $\%, 1$ $\mathrm{wt} \%, 2 \mathrm{wt} \%$ and $5 \mathrm{wt} \%$ ) were synthesized.

To verify the chemical bonding of PIM component within the PDMS network, a leaching experiment was carried out for the synthesized PDMS-PIM materials. Materials including PDMS-PIM1, PDMS-PIM2, PDMSPIM5 and PDMS-PIM*1 (This sample is synthesized by using PIM45 instead of PIM45-vinyl. It should not have chemical bonding between PIM component and PDMS network.) were immersed in PBS solution or deuterated $\mathrm{H}_{2} \mathrm{O}$ to assess if the PIM component is released from the PDMS-PIM material. PIM concentrations in PBS solution were calibrated and calculated by UV-vis spectroscopy (Fig. S2). PIM component in $\mathrm{D}_{2} \mathrm{O}$ was characterized by ${ }^{1} \mathrm{H}$ NMR spectrum (Fig. S3).

The release of PIM component from PDMS-PIM in PBS was observed over 5 cycles, indicating a sustained release of antimicrobial compound (Fig. 1A: $1 \mathrm{~h}$ per cycle and 1B: 1 day per cycle). Semi-quantitative assay further showed an initial burst of PIM component in the first two cycles followed by a gradual and sustained release up to five cycles (Fig. 1). A cumulative $5.6 \%$ of PIM component was released from the PDMS-PIM5 material in contrast to $90 \%$ release for PDMS-PIM*1 material. It is clear that the PDMS-PIM materials synthesized with PIM45-vinyl have much slower release profiles as compared with the material synthesized with PIM45 (without vinyl end groups) and this suggests that the chemical bonds were formed between PIM component and PDMS framework through hydrosilylation reaction (Fig. S4). The eluted solution and the recovered PDMSPIM biomaterial (after multiple cycles of PIM elution) exhibit antimicrobial activity (Fig. 1C, D). Furthermore, $\mathrm{D}_{2} \mathrm{O}$ solutions in which PDMS-PIM1 and PDMS-PIM2 materials were submerged contained dissolved PIM component lacking vinyl groups, as characterized by ${ }^{1} \mathrm{H}$ NMR spectroscopy. A minor amount of PIM component released from PDMS-PIM5 material contained the vinyl functional group (Fig. S3). These results indicate that the hydrosilylation reaction of PIM45-vinyl is

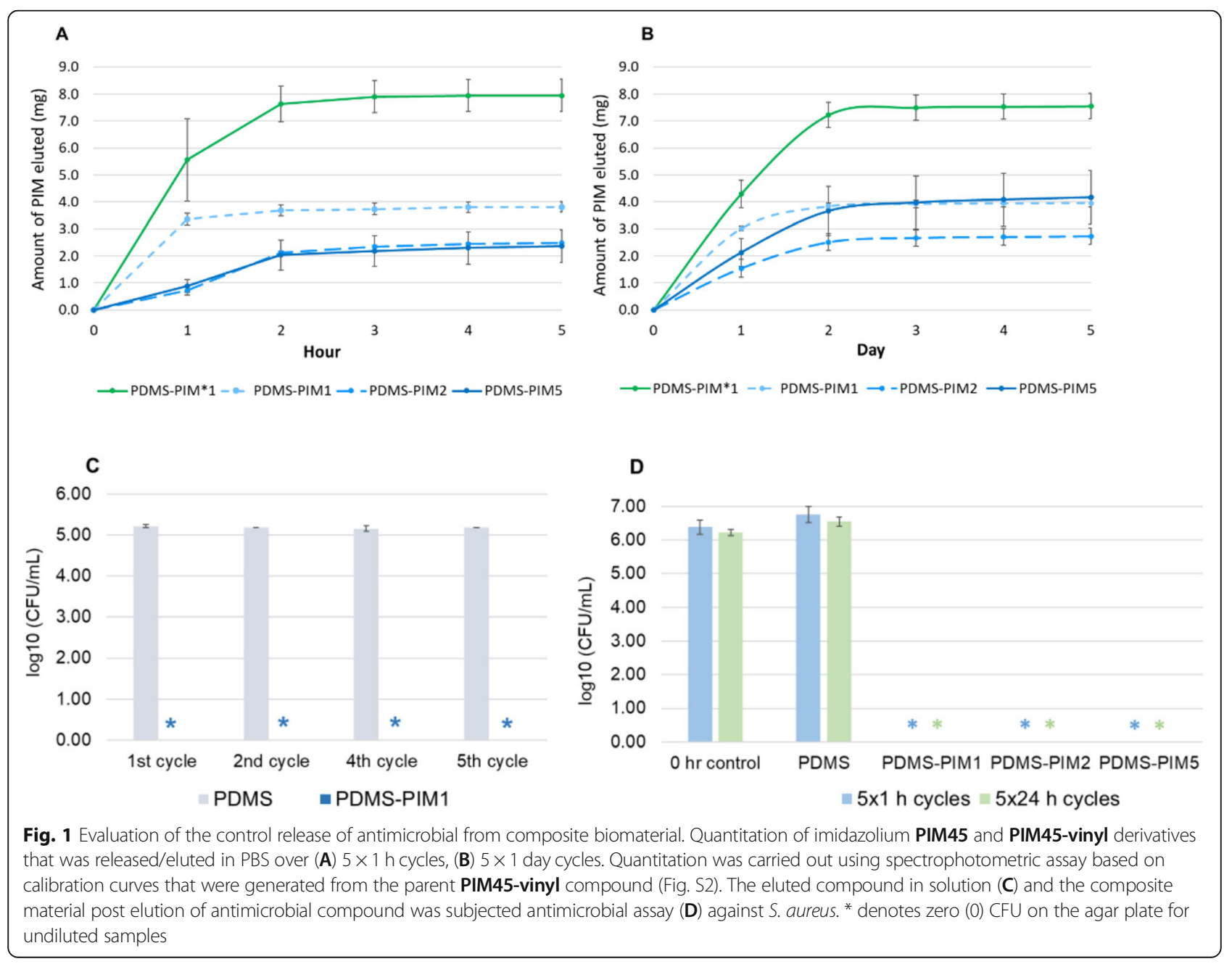


complete and all the PIM45 is chemically bonded in materials with 1 and 2 wt\% PIM45-vinyl loading, but incomplete in materials with 5 wt\% PIM45-vinyl loading. In PDMS-PIM1 and PDMS-PIM2 materials, the PIM leaching may result from hydrolysis of $\mathrm{Si}-\mathrm{O}$ bonds in the PDMS framework, which was also verified by ${ }^{1} \mathrm{H}-\mathrm{NMR}$ spectroscopy (Figs. S4, S5).

Our observation confirms that modifying the end group of an antimicrobial compound can be used to fabricate composite biomaterials. The resulting composite material integrates and retains the antimicrobial component in a manner which allows gradual and sustained release of the antimicrobial compound.

Surface topology of the biomaterial was determined using both FESEM and the AFM methodology. Surface roughness analysis on AFM showed that the root mean square (Rq) of the PDMS surface becomes even smoother upon inclusion of PIM45-vinyl (Fig. 2). Data from AFM corroborates with the SEM images which show that the surface of PDMS and its PDMS-PIM materials (PDMS-PIM1 and PDMS-PIM5) are smooth. The surface of PDMS-PIM materials are smooth and intrinsically hydrophobic. The contact angle measurements indicated that the surfaces of the PDMS-PIM biomaterials are generally less hydrophobic as compared with PDMS (Fig. 2C). Increasing the amount of integrated polymeric antimicrobial compound, which is hydrophilic in nature, decreases the hydrophobicity of the surface.

The surface antimicrobial property of the fabricated biomaterial was evaluated using modified Japanese Industrial Standard protocol [23]. The microbes (in neat media) were exposed to composite PDMS-PIM for $24 \mathrm{~h}$. The data revealed that PDMS-PIM0.5 and PDMSPIM1 have potent biocidal activity against E. coli, S. aureus and C. albicans (Fig. 3A). The time killing assay performed with PDMS-PIM1 and PDMS-PIM5 to assess the shortest exposure time needed to eliminate microbes on the surface of PDMS material. We found that the bactericidal property of PDMS-PIM1 was effective within $30 \mathrm{~s}$ of microbial exposure (Fig. 3B), exhibiting complete eradication of the microbes (single inoculation). In addition, PDMS-PIM materials used in this test have been stored at room temperature for up to 6 months.

The surface antimicrobial assay was repeated over longer durations to study the stability and durability of the PDMS-PIM biomaterial [26, 30]. The antimicrobial PDMS-PIM disks were challenged with respective microbes every $48-72 \mathrm{~h}$. The study was terminated at 45 days for single culture while three time points at 15 days, 30 days and 60 days were recorded for co-culture assay. In single culture assay, we observed complete bactericidal effect up to 45 days for PDMS-PIM biomaterials against both $S$. aureus and C. albicans. In contrast, the unmodified PDMS biomaterial exhibited colonization at $10^{11} \mathrm{CFU} / \mathrm{mL}$ and $10^{10} \mathrm{CFU} / \mathrm{mL}$ for $S$. aureus and $C$. albicans respectively (Table S1). Since multi microorganism infections and colonization is widespread in healthcare settings, we initiated a $S$. aureus and C. albicans co-culture in the laboratory and tested it on the composite PDMS-PIM materials (Fig. 4A). Microbes in this co-culture was identified by the phenotypic characteristics of the colonies. S. aureus forms golden colored colonies that distinguishes from the white colonies formed by C. albicans. PDMS-PIM1, PDMS-PIM2 and PDMS-PIM5 materials could fully eradicate co-cultured bacterial and fungal colonization after 15 days in culture. At the 30 day time point, reduction in antimicrobial potency was observed for PDMS-PIM1 against both $S$. aureus and C. albicans. While PDMS-PIM2 exhibited moderate efficacy against $C$. albicans but fully eradicated $S$. aureus (Fig. 4 A), a dose dependent phenomena. It is noteworthy that PDMS-PIM5 eliminated both $S$. aureus and $C$. albicans even after 60 days in co-culture, demonstrating excellent durability. Such qualities make PDMSPIM5 suited for practical medical applications, especially as long-term indwelling urinary catheters which typically could remain in place for more than 30 days.

To evaluate the reusability of the composite biomaterial, the PDMS-PIM materials recovered on Day 15 of co-culture incubation were subjected to a further $2 \mathrm{cy}$ cles (15 days / cycle) of co-culture incubation (Fig. 4B). We found that PDMS-PIM5 could last for 3 cycles of 15 days each ( 45 days in total) with washing and rinsing carried out every 15th day. Thus, PDMS-PIM5 exhibited stable antimicrobial properties after 3 cycles of periodical rinsing or washing. Reuse of the recovered PDMS-PIM material also confirmed that PDMS-PIM5 could effectively eradicate colonization of bacteria in cocultures.

In addition, the mechanical strength and mechanical properties of the material have been evaluated for the potential use as a catheter. Tensile strength/stress testing experiments indicate that the mechanical property of the PDMS material was not altered with incorporation of PIM45-vinyl (Table 1). Furthermore, the data also shows that the tensile strength remained unaltered with the extended durability. It is noteworthy that the tested material did not break/fracture during the experimental procedures $[14,31]$. Statistical analysis ( $t$-test, 2 tailed homoscedatic) Student's $t$-test between control and composite materials (pristine or used for either 45 Days or 60 Days) and with regards to load, tensile strength and modulus is shown in Table S2.

In order to assess the potential value of our biomaterial in medical device fabrication, the biomaterial was tested for its antimicrobial efficacy on clinical isolates 


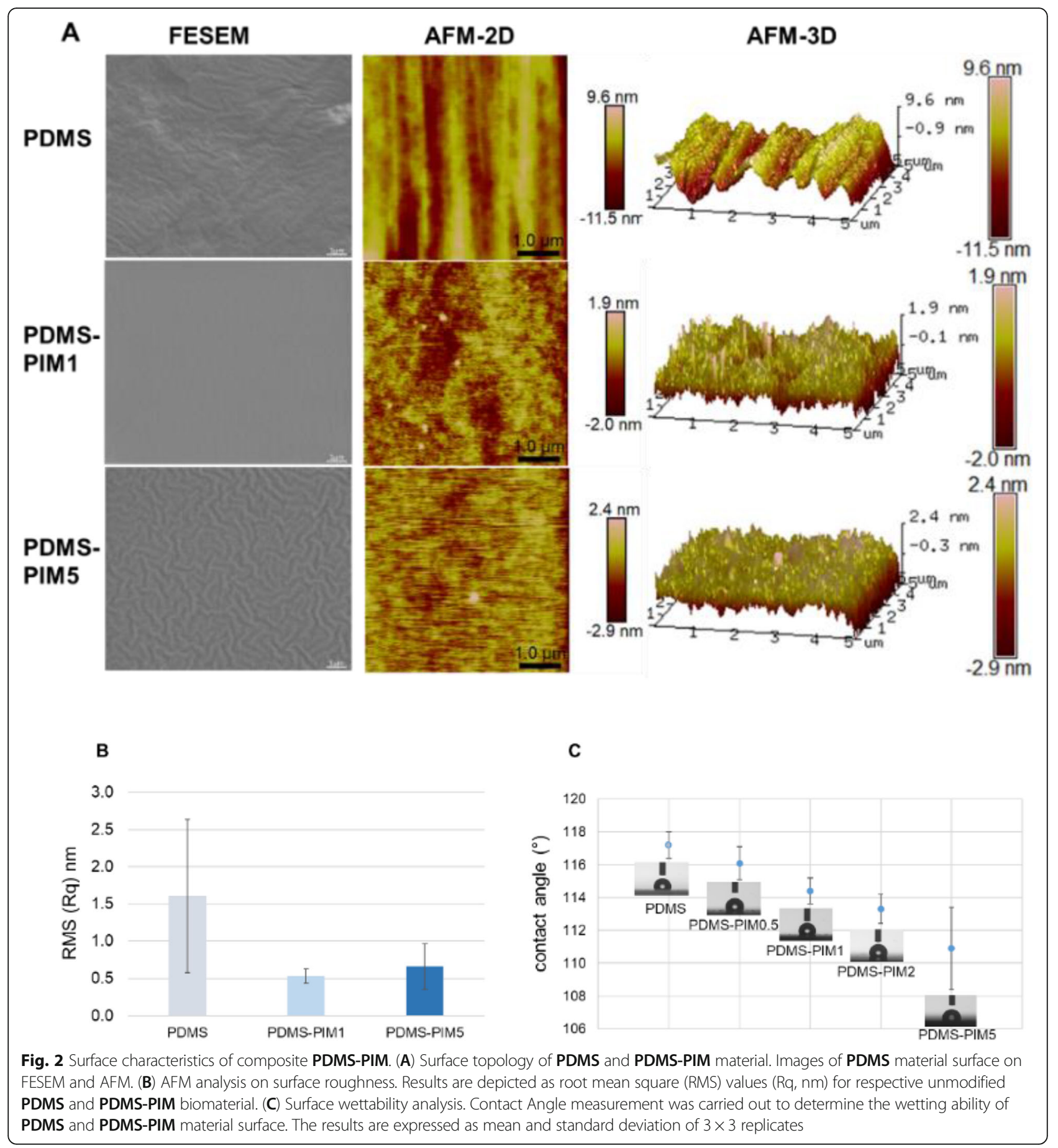

that are prevalent in hospital acquired infections (HAI). These microbes represent various antibiotic resistant (MDR) species often related to nosocomial infections in healthcare settings, especially in CAUTI. We found that PDMS-PIM1 inhibited the growth of all the clinical isolates. These microbes represent various MDR species often related to nosocomial infections in healthcare settings, especially in CAUTI. The acronym ESKAPE comprise of six nosocomial pathogens that exhibit multidrug resistance and virulence, namely Enterococcus faecium, Staphylococcus aureus, Klebsiella pneumoniae, Acinetobacter baumannii, Pseudomonas aeruginosa, and Enterobacter spp. Continual use of antibiotics has aggravated the emergence of MDR and extensively drug resistant (XDR) bacteria, that render even the most effective drugs ineffective. Extended spectrum $\beta$-lactamase (ESBL) and carbapenemase producing Gram negative bacteria have emerged as a recalcitrant therapeutic challenge. 


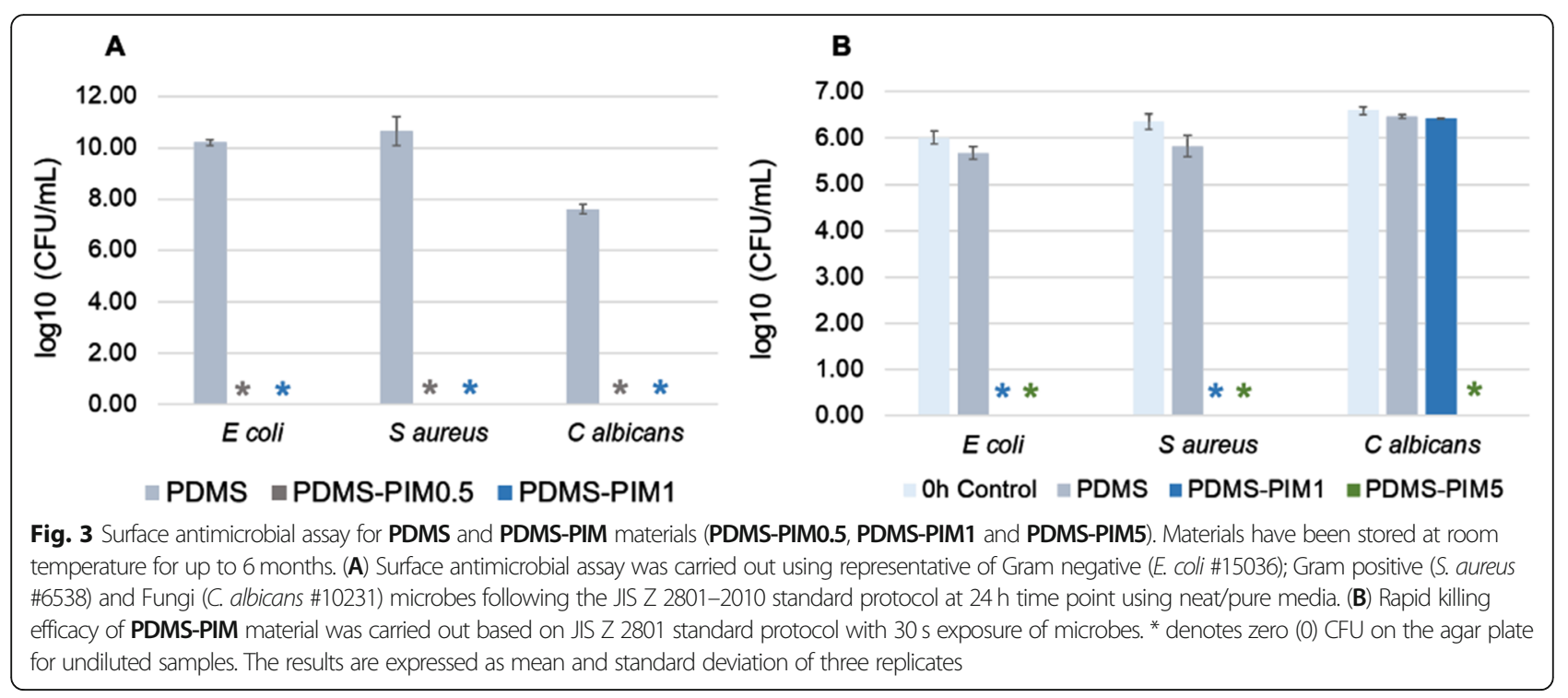

These MDR microbes are the most notorious group of bacteria that pose a serious global health threat [32, 33]. We found that our antimicrobial biomaterials (PDMSPIM1) inhibited the growth of all the ESKAPE group of clinical isolates as well as MRSA and Vancomycinresistant Enterococcus (VRE) pathogens (Fig. 5) and respective Candida $s p$ of clinical isolates.

Microbial colonization and formation of biofilm have become the major threat that leads to emergence of drug resistant microbes. Often, antimicrobial resistance is enhanced by the colonization of microbes and formation of biofilms. We formed biofilms on polystyrene, PDMS, low density polyethylene (tube) and glass cover slips and subsequently exposed these biofilms to PDMS-PIM1 material [14, 25, 34]. We found that PDMS-PIM1 could effectively eradicate biofilm colonies on all the surfaces. A time dependent assay showed that biofilm colonies on glass cover slips were eradicated within $1 \mathrm{~h}$ of contact with PDMS-PIM composite material (Fig. 6A). Biofilm formed in a tube could be eliminated by the eluate from $100 \mathrm{mg}$ of PDMS-PIM1 material in $10 \mathrm{ml}$ PBS solution (Fig. 6B) [22].

Microbial adhesion onto a surface is the initial stage of any pathogenesis, which subsequently leads to formation of the difficult to treat biofilms. PDMS, being inherently hydrophobic, exhibits an inherent antifouling property as a biomaterial as it does not support adherence of microbes to its surface. Inclusion of the PIM antimicrobial compound further enhances the antifouling and subsequently biocidal/antibiofilm properties of the PDMSPIM material, thus fulfilling the desired properties of an antimicrobial biomaterial.

Assessment of the materials' biocompatibility, comprising hemocompatibility and cytocompatibility assays,

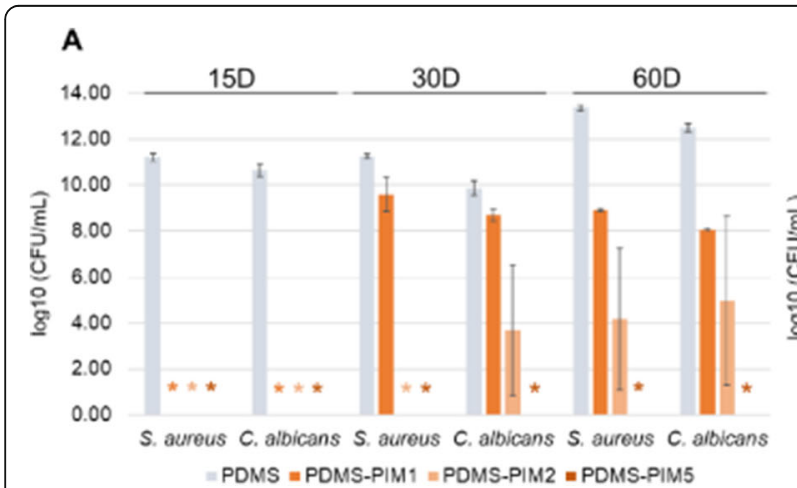

B

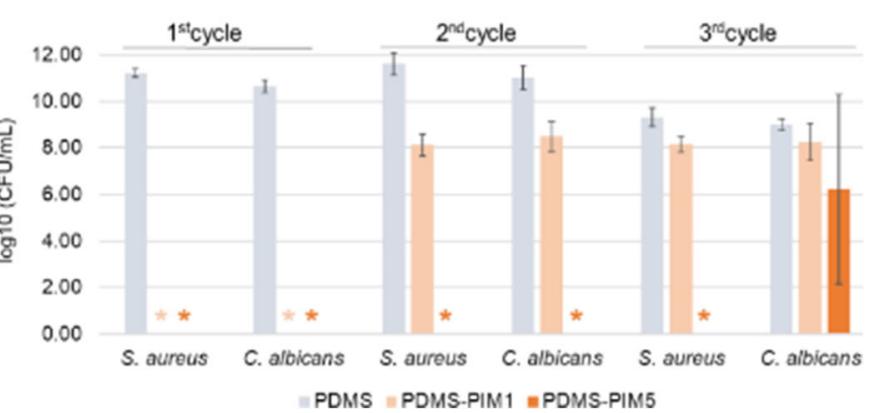

Fig. 4 (A) Durability of PDMS-PIM biomaterial in microbial co-culture with continuous challenge -inoculation at 48-72 $\mathrm{h}$ interval. The biomaterial was challenged with microbes (S. aureus and C. albicans) at 48-72 h intervals. The antimicrobial potency evaluation was carried out at 15 days, 30 days and 60 days endpoint. (B) Recycle/Reusable potential of recovered PDMS-PIM disk. Each cycle indicates a 15 days period that the biomaterials were challenged with microbial at $48-72 \mathrm{~h}$ interval, periodically, before being assessed for the antimicrobial potency. The results are expressed as mean and standard deviation of three replicates. * indicates zero (0) CFU on the agar plate for undiluted samples 
Table 1 Tensile strength and modulus of PDMS materials

\begin{tabular}{|c|c|c|c|}
\hline Materials & Modulus (Automatic Young's) [MPa] & Maximum Force $[\mathrm{N}]$ & Tensile stress at Maximum Force $[\mathrm{MPa}]$ \\
\hline \multicolumn{4}{|c|}{ As synthesized } \\
\hline PDMS & $1.78 \pm 0.35$ & $13.83 \pm 1.94$ & $0.51 \pm 0.07$ \\
\hline PDMS-PIM1 & $1.38 \pm 0.38$ & $10.30 \pm 2.34$ & $0.37 \pm 0.11$ \\
\hline PDMS-PIM5 & $1.11 \pm 0.28$ & $10.39 \pm 1.55$ & $0.37 \pm 0.04$ \\
\hline \multicolumn{4}{|c|}{45 Days of Microbial Exposure } \\
\hline PDMS & $1.79 \pm 0.06$ & $14.13 \pm 4.20$ & $0.43 \pm 0.13$ \\
\hline PDMS-PIM1 & $1.78 \pm 0.25$ & $11.73 \pm 1.73$ & $0.36 \pm 0.06$ \\
\hline PDMS-PIM5 & $1.31 \pm 0.20$ & $13.34 \pm 2.64$ & $0.42 \pm 0.07$ \\
\hline \multicolumn{4}{|c|}{60 Days of Microbial Exposure } \\
\hline PDMS & $1.91 \pm 0.02$ & $14.83 \pm 2.06$ & $0.40 \pm 0.05$ \\
\hline PDMS-PIM1 & $1.76 \pm 0.45$ & $11.24 \pm 1.06$ & $0.39 \pm 0.03$ \\
\hline PDMS-PIM5 & $1.28 \pm 0.09$ & $10.27 \pm 2.28$ & $0.39 \pm 0.03$ \\
\hline
\end{tabular}

$[27,28]$ was carried out to evaluate the safety of PDMSPIM biomaterial for potential medical application. A 10 mg sample of PDMS-PIM5 material exhibited 1.44 \pm $0.56 \%$ hemolysis, while PDMS-PIM1 showed $0.3 \pm$ $0.26 \%$ hemolysis (Fig. 7A). Cell viability assay using murine derived fibroblast L929 cells (Fig. 7B) also proved that our composite biomaterial is non-toxic to mammalian cells in culture. Hence, we found that the PDMSPIM materials are biocompatible supported by both the hemo- and cyto-compatibility assessments.

\section{Discussions}

Catheter associated infections (CAI) pose a serious health threat and remains a challenge in healthcare settings worldwide. Patients requiring indwelling catheters, especially urinary catheters, often get infections within days of catheter use. Though antimicrobial-coated catheters are currently available, their efficacy in clinics are still far from satisfactory [6-8]. In this study, PDMS based biomaterial containing an antimicrobial imidazolium polymer demonstrated several key characteristics which may indicate the potential application of these materials to mitigate catheter associated infections. (1) PDMS-PIM biomaterial exhibited potent antimicrobial activity and fast killing property with $30 \mathrm{~s}$ of exposure to the microbes resulting in complete eradication of the microbes. The material demonstrated long lasting activity that could inhibit the colonization of both $S$. aureus and $C$. albicans for at least 45 days in culture. Eradication of microbial colonization on a subsequent inoculation of the recovered and reused material further

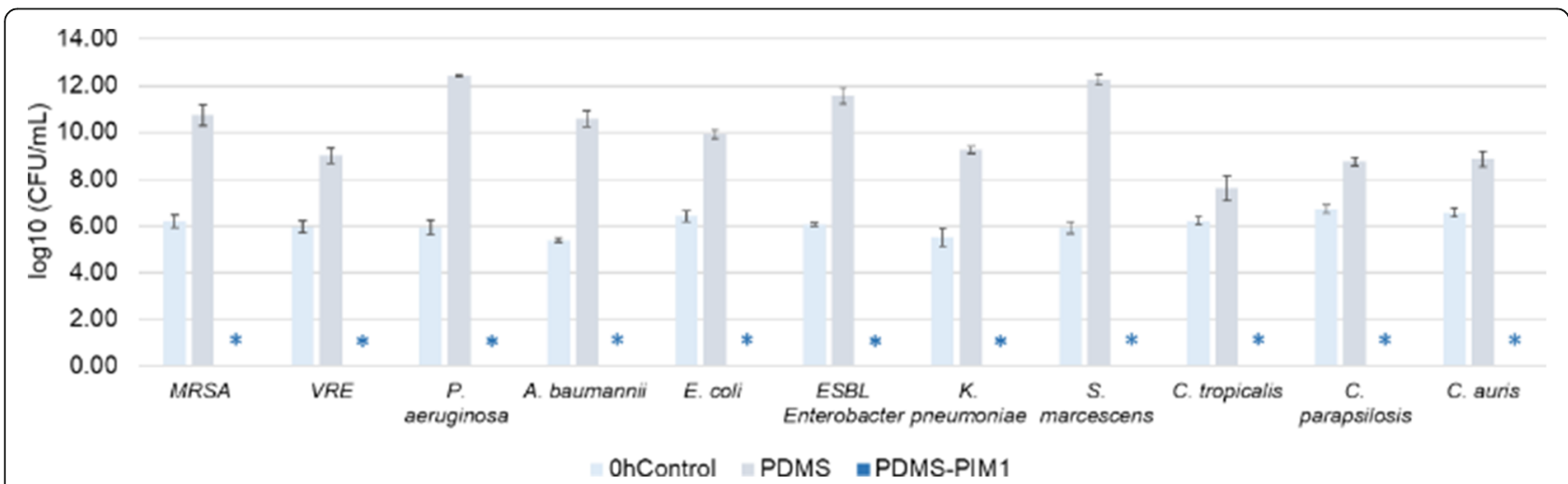

Fig. 5 Efficacy of antimicrobial activity of PDMS-PIM1 on clinical pathogens. Potency of polymer antimicrobial PDMS-PIM1 against clinical pathogens (Enterococcus faecium-VR Enterococcus; Staphylococcus aureus-MRSA; Klebsiella pneumoniae, Acinetobacter baumannii, Pseudomonas aeruginosa, Enterobacter species-ESBL Enterobacter, S. marcescens, E. coli, C. parapsilosis, C. tropicalis and C. auris) was evaluated following JIS Z 2801-2010 protocol. The results are expressed as mean and standard deviation of three replicates. * denotes zero (0) CFU on the agar plate for undiluted samples 


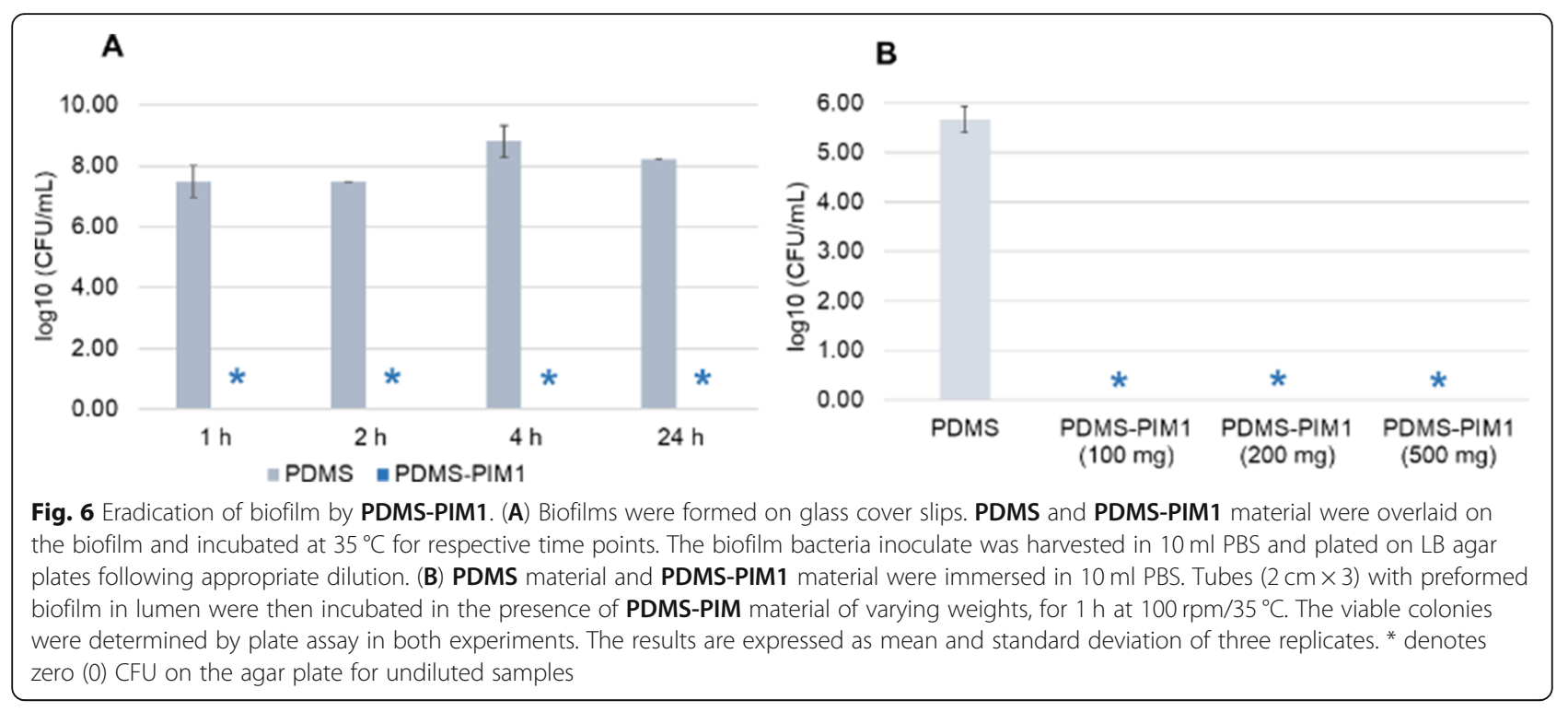

confirm that our PDMS-PIM material could be recycled for usage. The PDMS-PIM material does not cause any change or adverse effect on the mechanical performance of the fabricated materials, either pristine or used products. The tensile property of the fabricated materials were also not altered with extended use, thus confirming their durability. (2) Controlled release of antimicrobial substance from a polymeric material has been shown to be useful in preventing planktonic as well as biofilm microbial growth on biomaterials [34-38]. It is a challenge to control the amount of antimicrobial compound that is coated, embedded or impregnated from the outset. In contrast, the method described herein results in a composite PDMS-PIM biomaterial casted with a well- defined amount of antimicrobial polymer that is fully integrated and chemically bonded in the material. The amount of antimicrobial compound in the biomaterials can be accurately tuned to fit the requirements and usage conditions of the resulting device. (3) PDMS-PIM materials continues to release active compounds over a prolonged period of time. The releasing process mainly involves hydrolysis of $\mathrm{Si}-\mathrm{O}$ bonds. Initial burst release was followed by subsequent sustained release of active antimicrobial compound. The release profile is demanded to curtail the biofilm formation and sustain continuous treatment [39]. (4) The ESKAPE family of MDR bacteria are the most notorious group of bacteria that pose a serious global health threat and account for

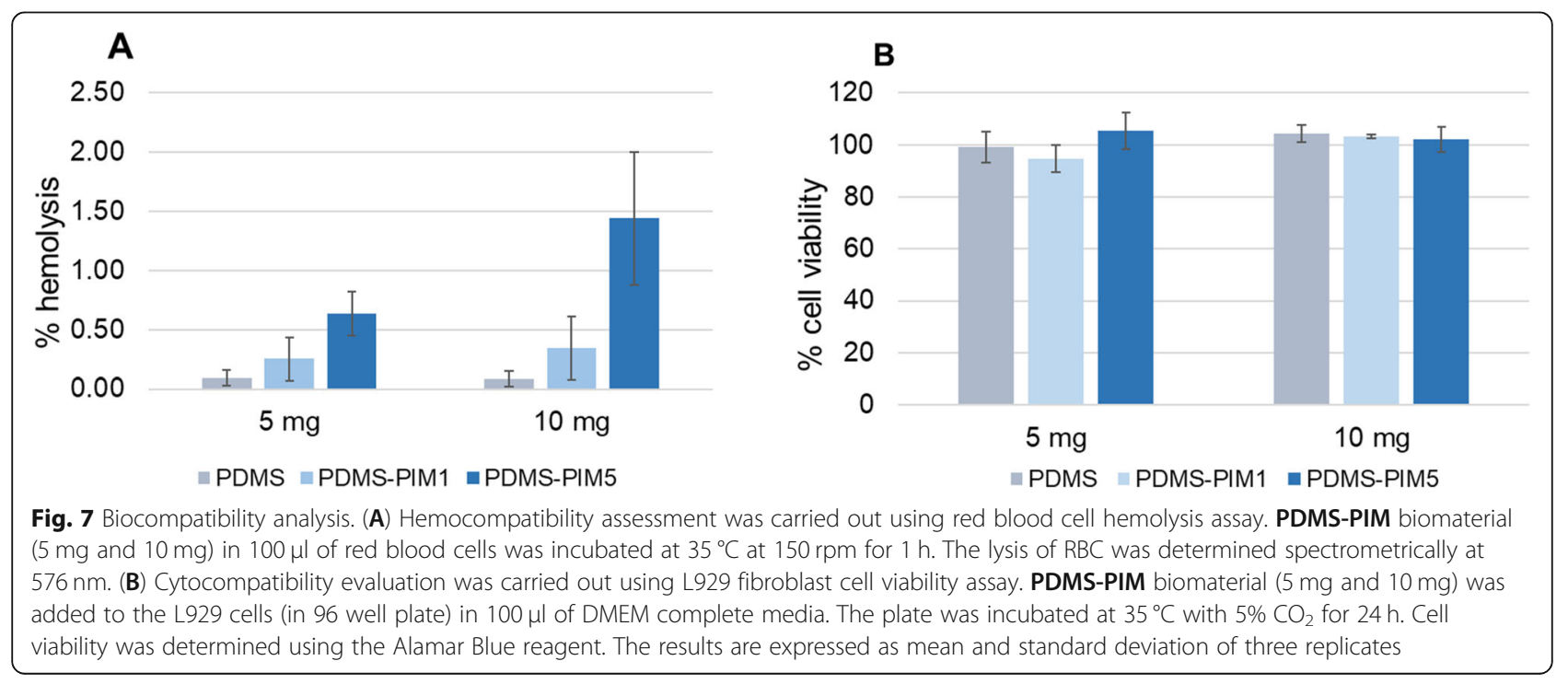


up to $87 \%$ of all hospital acquired infections [40]. This family of bacteria has been listed as "high priority" under the WHO priority listing [41-45]. It is noteworthy that PDMS-PIM biomaterials could efficiently and effectively inhibit the colonization of all the members of ESKAPE group of pathogens as well as the WHO priority pathogens such MRSA and VRE. The susceptibility of these drug resistant bacteria towards PDMS-PIM further endorse that our composite biomaterials in the form of catheters could prove beneficial in preventing catheterassociated infections especially in CAUTI. In addition, the composite PDMS-PIM biomaterial is biocompatible and exhibits stable antimicrobial activity up to at least 6 months storage at room temperature.

\section{Conclusions}

We have fabricated a biocidal PDMS-PIM biomaterial that shows sustained release of the polymeric antimicrobial. Our approach will prove beneficial in fabricating antimicrobial biomaterials for manufacturing devices for medical and healthcare applications. Compared to available products that rely on coating or impregnation to introduce antimicrobial components, the PDMS-PIM biomaterial presented in this study will be easier to manufacture. No additional steps to coat or impregnate the material with antimicrobial compounds are needed. In addition, the amount of antimicrobial compounds to be incorporated for fabrication can be regulated during the one-time casting process. The new material demonstrated excellent antimicrobial activity, being able to efficiently inhibit or eliminate the colonization of both Gram positive (S. aureus) and Gram negative (E. coli) bacteria as well as fungi (C. albicans) in single or coculture experiments. Furthermore, PDMS-PIM composite material effectively functioned as a bactericidal and fungicidal surface against multidrug resistant clinical isolates including the ESKAPE family of pathogens. Stability and durability studies showed that the new material could withstand prolonged incubation with continuous challenge with microbes - up to 60 days against single culture bacterial or fungi or their co-culture. Hence, the PDMS-PIM materials have the potential to be used for antifouling/antibiofilm urinary catheters that require long-term continuous utilization.

\section{Supplementary Information}

The online version contains supplementary material available at https:/doi. org/10.1186/s40824-021-00235-5.

Additional file 1.

\section{Acknowledgements}

This work was funded by the Institute of Bioengineering and Bioimaging, Biomedical Research Council, Agency for Science, Technology and Research, Singapore. The authors acknowledge the contribution of Dr. Shujun Gao,
NanoBio Lab, A*Star for the fresh murine blood sample for hemolysis assays. The clinical pathogens were provided by Dr. Shawn Vasoo of the Infectious Diseases Research Laboratory, the National Centre for Infectious Diseases and Tan Tock Seng Hospital.

\section{Authors' contributions}

A-A., S.P-T., D. S. W-L., S. P-C., G. S-Y., Y-Z., conceived and designed the analyses. Y-Z., acquired funding. A-A., S.P-T., D. S. W-L., S. P-C., G. S-Y., Y-Z., collected the data, performed the analyses and wrote the paper. C.W-B., D. S-Y., carried out the mechanical strength measurements, analysed and computed the results. The author(s) read and approved the final manuscript.

\section{Funding}

This work was funded by the Institute of Bioengineering and Bioimaging, Biomedical Research Council, Agency for Science, Technology and Research, Singapore.

Availability of data and materials

All data are available on request.

\section{Declarations}

Ethics approval and consent to participate

Do not apply.

\section{Consent for publication}

All authors agree with the publication of this research.

\section{Competing interests}

The authors declare they have no competing interests.

\section{Author details}

${ }^{1}$ Institute of Bioengineering and Bioimaging, 31 Biopolis Way, The Nanos, Singapore 138669, Singapore. ${ }^{2}$ Molecular Engineering Lab, Institute of Molecular and Cell Biology, 61 Biopolis Drive, The Proteos, Singapore 138673, Singapore.

Received: 8 July 2021 Accepted: 26 September 2021

Published online: 21 October 2021

\section{References}

1. Knight GM, Glover RE, McQuaid CF, Olaru ID, Gallandat K, Leclerc QJ, et al. Antimicrobial resistance and COVID-19: intersections and implications. eLife. 2021;10:e64139. https://doi.org/10.7554/eLife.64139.

2. Pelfrene $\mathrm{E}$, Botgros $\mathrm{R}$, Cavaleri M. Antimicrobial multidrug resistance in the era of COVID-19: a forgotten plight? Antimicrob Resist Infect Control. 2021; 10(1):21. https://doi.org/10.1186/s13756-021-00893-z.

3. Weiner LM, Webb AK, Limbago B, Dudeck MA, Patel J, Kallen AJ, et al. Antimicrobial-resistant pathogens associated with healthcare-associated infections: summary of data reported to the national healthcare safety network at the centers for disease control and prevention, 2011-2014. Infect Control Hosp Epidemiol. 2016;37(11):1288-301. https://doi.org/10.1017/ice.2 016.174.

4. Andersen MJ, Flores-Mireles AL. Urinary catheter coating modifications: the race against catheter-associated infections. Coatings. 2020;10(1):23. https:// doi.org/10.3390/coatings10010023.

5. Ahonen M, Kahru A, Ivask A, Kasemets K, Kõljalg S, Mantecca P, et al. Proactive approach for safe use of antimicrobial coatings in healthcare settings: opinion of the COST action network AMiCl. Int J Environ Res Public Health. 2017;14(4):366. https://doi.org/10.3390/ijerph14040366.

6. Riley DK, Classen DC, Stevens LE, Burke JP. A large randomized clinical trial of a silver-impregnated urinary catheter: lack of efficacy and staphylococcal superinfection. Am J Med. 1995;98(4):349-56. https://doi.org/10.1016/S00029343(99)80313-1.

7. Srinivasan A, Karchmer T, Richards A, Song X, Perl TM. A prospective trial of a novel, silicone-based, silver-coated Foley catheter for the prevention of nosocomial urinary tract infections. Infect Control Hosp Epidemiol. 2006; 27(1):38-43. https://doi.org/10.1086/499998.

8. Menezes FG, Correa L, Medina-Pestana JO, Aguiar WF, Camargo LFA. A randomized clinical trial comparing Nitrofurazone-coated and uncoated 
urinary catheters in kidney transplant recipients: results from a pilot study. Transpl Infect Dis. 2019;21(2):e13031. https://doi.org/10.1111/tid.13031.

9. Hodgkinson V, Petris MJ. Copper homeostasis at the host-pathogen interface. J Biol Chem. 2012;287(17):13549-55. https://doi.org/10.1074/jbc. R111.316406.

10. Zhu Z, Wang Z, Li S, Yuan X. Antimicrobial strategies for urinary catheters. J Biomed Mater Res A. 2019;107(2):445-67. https://doi.org/10.1002/jbm.a.36561.

11. Zhang J, Chen Y, Brook MA. Facile functionalization of PDMS elastomer surfaces using thiol-ene click chemistry. Langmuir. 2013;29(40):12432-42. https://doi.org/10.1021/la403425d.

12. Milo S, Thet NT, Liu D, Nzakizwanayo J, Jones BV, Jenkins ATA. An in-situ infection detection sensor coating for urinary catheters. Biosens Bioelectron. 2016;81:166-72. https://doi.org/10.1016/j.bios.2016.02.059.

13. Bayston R, Fisher LE, Weber K. An antimicrobial modified silicone peritoneal catheter with activity against both gram-positive and gram-negative bacteria. Biomaterials. 2009;30(18):3167-73. https://doi.org/10.1016/j.bioma terials.2009.02.028.

14. Fisher LE, Hook AL, Ashraf W, Yousef A, Barrett DA, Scurr DJ, et al. Biomaterial modification of urinary catheters with antimicrobials to give long-term broadspectrum antibiofilm activity. J Control Release. 2015;202: 57-64. https://doi.org/10.1016/j.jconrel.2015.01.037.

15. Thomé IPS, Dagostin VS, Piletti R, Pich CT, Riella HG, Angioletto E, et al. Bactericidal low density polyethylene (LDPE) urinary catheters: microbiological characterization and effectiveness. Mater Sci Eng C. 2012; 32(2):263-8. https://doi.org/10.1016/j.msec.2011.10.027.

16. Anjum S, Gupta B. Bioengineering of functional nanosilver nanogels for smart healthcare systems. Glob Chall. 2018;40(10):1800044. https://doi.org/1 0.1002 /gch2.201800044.

17. Lim K, Chua RR, Bow H, Tambyah PA, Hadinoto K, Leong SS. Development of a catheter functionalized by a polydopamine peptide coating with antimicrobial and antibiofilm properties. Acta Biomater. 2015;15:127-38. https://doi.org/10.1016/j.actbio.2014.12.015.

18. Lim K, Saravanan R, Chong KKL, Goh SHM, Chua RRY, Tambyah PA, et al. Anhydrous polymer-based coating with sustainable controlled release functionality for facile, efficacious impregnation, and delivery of antimicrobial peptides. Biotechnol Bioeng. 2018;115(8):2000-12. https://doi. org/10.1002/bit.26713.

19. Liu L, Wu H, Riduan SN, Ying JY, Zhang Y. Short imidazolium chains effectively clear fungal biofilm in keratitis treatment. Biomaterials. 2013;34(4): 1018-23. https://doi.org/10.1016/j.biomaterials.2012.10.050.

20. Liu L, Huang Y, Riduan SN, Gao S, Yang Y, Fan W, et al. Main-chain imidazolium oligomer material as a selective biomimetic antimicrobial agent. Biomaterials. 2012;33(33):8625-31. https://doi.org/10.1016/j.biomateria Is.2012.08.006

21. Salernitano E, Migliaresi C. Composite materials for biomedical applications: a review. J Appl Biomater Biomech. 2003;1(1):3-18.

22. Malcolm RK, McCullagh SD, Woolfson AD, Gorman SP, Jones DS, Cuddy J. Controlled release of a model antibacterial drug from a novel selflubricating silicone biomaterial. J Control Release. 2004;97(2):313-20. https:// doi.org/10.1016/j.jconrel.2004.03.029.

23. Japanese Industrial Standard JIS Z 2801. Test for antibacterial activity and efficacy. www.jsa.or.jp. Japanese Standards Association. Tokyo. Japan. 2010

24. Merritt JH, Kadouri DE, O'Toole GA. Growing and analyzing static biofilms. Curr Protoc Microbiol. 2005;Chapter 1:Unit 1B.1. https://doi.org/10.1002/ 9780471729259.mc01b01s00.

25. Mandakhalikar KD, Rahmat JN, Chiong E, Neoh KG, Shen L, Tambyah PA. Extraction and quantification of biofilm bacteria: method optimized for urinary catheters. Sci Rep. 2018;8(1):8069. https://doi.org/10.1038/s41598-01 8-26342-3.

26. Zhang S, Wang L, Liang X, Vorstius J, Keatch R, Corner G, et al. Enhanced antibacterial and anti-adhesive activities of silver-PTFE nanocomposite coating for urinary catheters. ACS Biomater Sci Eng. 2019;5(6):2804-14. https://doi.org/10.1021/acsbiomaterials.9b00071.

27. Chamsaz EA, Mankoci S, Barton HA, Joy A. Nontoxic cationic coumarin polyester coatings prevent pseudomonas aeruginosa biofilm formation. ACS Appl Mater Interfaces. 2017;9(8):6704-11. https://doi.org/10.1021/acsami. 6b12610.

28. Pendleton JN, Gorman SP, Gilmore BF. Clinical relevance of the ESKAPE pathogens. Expert Rev Anti-Infect Ther. 2013;11(3):297-308. https://doi.org/1 0.1586/eri.13.12.
29. Liu K, Shen X, Bai S, Conrad Zhang ZC. Stable discrete Pt (0) in crown ether with ultra-high hydrosilylation activity. ChemCatChem. 2020;12(1):267-72. https://doi.org/10.1002/cctc.201901577.

30. Zhou C, Wu Y, Thappeta KRV, Subramanian JTL, Pranantyo D, Kang ET, et al. In vivo anti-biofilm and anti-bacterial non-leachable coating thermally polymerized on cylindrical catheter. ACS Appl Mater Interfaces. 2017;9(41): 36269-80. https://doi.org/10.1021/acsami.7b07053.

31. Cruz-Félix AS, Santiago-Alvarado A, Márquez-García J, González-García J. PDMS samples characterization with variations of synthesis parameters for tunable optics applications. Heliyon. 2019;5(12):e03064. https://doi.org/10.1 016/j.heliyon.2019.e03064.

32. Mulani MS, Kamble EE, Kumkar SN, Tawre MS, Pardesi KR. Emerging strategies to combat ESKAPE pathogens in the era of antimicrobial resistance: a review. Front Microbiol. 2019;10:539. https://doi.org/10.3389/ fmicb.2019.00539.

33. Ricardo SIC, Anjos IIL, Monge N, Faustino CMC, Ribeiro IAC. A glance at antimicrobial strategies to prevent catheter-associated medical infections. ACS Infect Dis. 2020;6(12):3109-30. https://doi.org/10.1021/acsinfecdis. 0c00526.

34. Francolini I, Vuotto C, Piozzi A, Donelli G. Antifouling and antimicrobial biomaterials: an overview. APMIS. 2017;125(4):392-417. https://doi.org/1 $0.1111 /$ apm.12675.

35. Dave RN, Joshi HM, Venugopalan VP. Novel biocatalytic polymer-based antimicrobial coatings as potential ureteral biomaterial: preparation and in vitro performance evaluation. Antimicrob Agents Chemother. 2011;55(2): 845-53. https://doi.org/10.1128/AAC.00477-10.

36. Liu H, Shukla S, Vera-González N, Tharmalingam N, Mylonakis E, Fuchs BB, et al. Auranofin releasing antibacterial and antibiofilm polyurethane intravascular catheter coatings. Front Cell Infect Microbiol. 2019;9:37. https:// doi.org/10.3389/fcimb.2019.00037.

37. Schierholz JM, Steinhauser H, Rump AF, Berkels R, Pulverer G. Controlled release of antibiotics from biomedical polyurethanes: morphological and structural features. Biomaterials. 1997;18(12):839-44. https://doi.org/10.1016/ S0142-9612(96)00199-8.

38. Riool M, de Breij A, Drijfhout JW, Nibbering PH, Zaat SAJ. Antimicrobial peptides in biomedical device manufacturing. Front Chem. 2017;5:63. https://doi.org/10.3389/fchem.2017.00063.

39. Wu P, Grainger DW. Drug/device combinations for local drug therapies and infection prophylaxis. Biomaterials. 2006;27(11):2450-67. https://doi.org/10.1 016/j.biomaterials.

40. Sievert DM, Ricks P, Edwards JR, Schneider A, Patel J, Srinivasan A, et al. National Healthcare Safety Network (NHSN) team and participating NHSN facilities. Antimicrobial-resistant pathogens associated with healthcareassociated infections: summary of data reported to the National Healthcare Safety Network at the Centers for Disease Control and Prevention, 20092010. Infect Control Hosp Epidemiol. 2013;34(1):1-14. https://doi.org/10.1 $086 / 668770$

41. Si Z, Lim HW, Tay MYF, Du Y, Ruan L, Qiu H, et al. A glycosylated cationic block poly( $\beta$-peptide) reverses intrinsic antibiotic resistance in all ESKAPE gram-negative bacteria. Angew Chem Int Ed. 2020;59(17):6819-26. https:// doi.org/10.1002/anie.201914304.

42. Wu B, Yang $X$, Yan M. Synthesis and structure-activity relationship study of antimicrobial auranofin against ESKAPE pathogens. J Med Chem. 2019; 62(17):7751-68. https://doi.org/10.1021/acs.jmedchem.9b00550.

43. Lin Q, Deslouches B, Montelaro RC, Di YP. Prevention of ESKAPE pathogen biofilm formation by antimicrobial peptides WLBU2 and LL37. Int J Antimicrob Agents. 2018;52(5):667-72. https://doi.org/10.1016/j.ijantimicag.2 018.04.019.

44. Lam SJ, O'Brien-Simpson NM, Pantarat N, Sulistio A, Wong EH, Chen YY, et al. Combating multidrug-resistant gram-negative bacteria with structurally nanoengineered antimicrobial peptide polymers. Nat Microbiol. 2016;1(11):16162. https://doi.org/10.1038/nmicrobiol.2016.162.

45. Hall T, Villapun V, Addison O, Webber M, Lowther M, Louth S, et al. A call for action to the biomaterial community to tackle antimicrobial resistance. Biomaterials Science. 2020;8(18):4951-74. https://doi.org/10.1039/D0BM01160F.

\section{Publisher's Note}

Springer Nature remains neutral with regard to jurisdictional claims in published maps and institutional affiliations. 